\title{
A MULTI-AGENT MPC ARCHITECTURE FOR DISTRIBUTED LARGE SCALE SYSTEMS
}

\author{
Valeria Javalera, Bernardo Morcego and Vicenç Puig \\ Advanced Control Systems Group, Institut de Robòtica i Informàtica Industrial, CSIC-UPC \\ C/. Llorens i Artigas, 4-6, 08028 Barcelona, Spain \\ vjavalera@iri.upc.edu, bernardo.morcego@upc.edu,vicenc.puig@upc.edu
}

\begin{abstract}
Keywords: Large scale systems, Multi agent systems, Distributed model predictive control, Reinforcement learning.
Abstract: In the present work, techniques of Model Predictive Control (MPC), Multi Agent Systems (MAS) and Reinforcement Learning (RL) are combined to develop a distributed control architecture for Large Scale Systems (LSS). This architecture is multi-agent based. The system to be controlled is divided in several partitions and there is an MPC Agent in charge of each partition. MPC Agents interact over a platform that allows them to be located physically apart. One of the main new concepts of this architecture is the Negotiator Agent. Negotiator Agents interact with MPC Agents which share control variables. These shared variables represent physical connections between partitions that should be preserved in order to respect the system structure. The case of study, in which the proposed architecture is being applied and tested, is a small drinking water network. The application to a real network (the Barcelona case) is currently under development.
\end{abstract}

\section{INTRODUCTION}

Large Scale Systems (LSS) are complex dynamical systems at service of everyone and in charge of industry, governments, and enterprises. The applications are wide. Examples of applications of LSS in continuous domains are: power networks, sewer networks, water networks, canal and river networks for agriculture, etc. Other examples of applications of LSS in discrete domain are traffic control, railway control, manufacturing industry, etc.

Experts in automatic control have developed many solutions for this kind of systems. However, the increase of automation of LSS renders problems with a noticeable increase in complexity. Such complexity is due to the size of the system to be controlled and the huge number of sensors and actuators needed to carry out the control. Additionally, LSS are composed of many interacting subsystems. Thus, LSS control is difficult to be implemented using a centralized control structure because of robustness and reliability problems and due to communication limitations. For all these reasons, distributed control schemes have been proposed over the last years.

One of the main problems of distributed control of LSS is how relations between system partitions are preserved. These relations could be, for example, pipes that connect two different control zones of a decentralized water transport network, or any other kind of connection between different control zones. When these connections represent control variables, the distributed control has to be consistent for both zones and the optimal value of these variables will have to accomplish a common goal.

The authors believe that this open problem in control theory can be solve by the combination of adequate control and computer science techniques, more precisely, the combination of Model Predictive control (MPC), Multi-Agent Systems (MAS), and Reinforcement Learning (RL).

Due to Distributed Control (DC) present the same philosophy of Distributed Artificial Intelligence (DAI), the idea is to apply MAS techniques and technology to DC problems as communication, coordination, need of adaptation (learning), autonomy and intelligence.

The use of RL in the negotiation process will allow to:

1) Make the process of negotiation adaptive; 2 ) Learn from its own experience; 3) Explicitly consider the whole problem of two goal-oriented agents; 3) Deal with a dynamical and uncertain environment; 4) Optimize with or without a model; 
5) Connect the process of negotiation whit the one of the control MPC, this because of compatibilities found between them.

Model Predictive Control (MPC), also known as receding horizon control, is a control technique widely used in industry (see (Qin \& Badwell, 2003) and (Camacho \& Bordons, 2004)) well suited for the control of continuous LSS. In MPC, the control input is obtained by solving a discrete-time optimal control problem over a given horizon, producing an optimal open-loop control input sequence. The first control in that sequence is applied. At the next sampling instant, a new optimal control problem is formulated and solved based on the new measurements.

The MPC control aim is to find actions $u_{k}, \ldots, u_{k+N_{\mathrm{c}}}$, such that after $N_{\mathrm{p}}$ steps, a sequence of predicted outputs $y$ approaches a set point $y^{*}$.

The theory of MPC is well developed; most aspects, such as stability, nonlinearity, and robustness, have been discussed in the literature (see, e.g., (Bemporad \& Morari, 1999) (Morari \& Lee, 1999). Besides, MPC is very popular in the process control industry because the actual control objectives and operating constraints can be represented explicitly in the optimization problem that is solved at each control instant.

Typically, MPC is implemented in a centralized fashion. The complete system is modelled, and all the control inputs are computed in one optimization problem.

The goal of the research described in this paper is to exploit the attractive features of MPC (meaningful objective functions and constraints) in a distributed implementation combining learning techniques to perform the negotiation of these variables in a cooperative Multi Agent environment and over a Multi Agent platform. All these ideas are the basis of the proposed architecture. A methodology for the application of the proposed architecture is also provided.

Organization of the paper is as follows: Section 2 introduces the problem to be solved and Section 3 describes known approaches to it based on distributed MPC. The formalization of the proposed architecture is given in Section 4. Section 5 and 6 are devoted to an application example and the results obtained. Finally, Section 7 presents the paper conclusions and Section 8 presents the current and further research that is being developed.

\section{THE PROBLEM}

In order to control a LSS in a distributed way, some assumptions have to be made on its dynamics, i.e. on the way the system behaves. Assume, first, that the system can be divided into $n$ subsystems, where each subsystem consists of a set of nodes and the interconnections between them. The problem of determining the partitions of the network is not addressed in this paper; instead the reader is referred to (Siljack, 1991). The set of partitions should be complete. This means that all system states and control variables should be included at least in one of the partitions.

Definition 1. System Partitions. $P$ is the set of system partitions and is defined by

$$
P=\left\{p_{1}, p_{2}, \ldots, p_{n p}\right\}
$$

where each system partition (subsystem) $p_{i}$ is described by a deterministic linear time-invariant model that is expressed in discrete-time as follows

$$
\begin{aligned}
& \mathbf{x}_{i}(k+1)=\mathbf{A}_{i} \mathbf{x}_{i}(k)+\mathbf{B}_{u, i} \mathbf{u}_{i}(k)+\mathbf{B}_{d, i} \mathbf{d}_{i}(k) \\
& \mathbf{y}_{i}(k)=\mathbf{C}_{i} \mathbf{x}_{i}(k)+\mathbf{D}_{u, i} \mathbf{u}_{i}(k)+\mathbf{D}_{d, i} \mathbf{d}_{i}(k)
\end{aligned}
$$

where variables $\mathbf{x}, \mathbf{y}, \mathbf{u}$ and $\mathbf{d}$ are the state, output, input and disturbance vectors, respectively; A, C, B and $\mathbf{D}$ are the state, output, input and direct matrix, respectively. Subindices $u$ and $d$ refer to the type of inputs the matrix model, either control inputs or disturbances.

Definition 2. Internal Variables. Internal variables are control variables that appear in the model of only one subsystem in the problem. The set of internal variables of one partition is defined by

$$
U=\left\{u_{1}, u_{2}, \ldots, u_{n u}\right\}
$$

Definition 3. Shared Variables. Shared variables are control variables that appear in the model of at least two subsystems in the problem. Their values should be consistent in the subsystems they appear, so they are also called negotiated variables. $V$ is the set of negotiated variables defined by

$$
V=\left\{v_{1}, v_{2}, \ldots, v_{n v}\right\}
$$

Each subsystem $i$ is controlled by an MPC controller using:

- $\quad$ the model of the dynamics of subsystem $i$ given by equation (2);

- $\quad$ the measured state $\mathbf{x}_{i}(k)$ of subsystem $i$; 
- the exogenous inputs $\mathbf{d}_{i}(k+1)$ of subsystem $i$ over a specific horizon of time;

As a result each MPC controller determines the values $\mathbf{u}_{i}(k)$ of subsystem $i$. The internal control variables are obtained directly by the MPC controller of this subsystem while the shared variables are proposed to be negotiated with the MPC controllers of the corresponding subsystem.

When there are no shared variables between subsystems, this control scheme is usually referred to as decentralized control. When the set of shared variables is not empty, it is usually referred to as distributed control. The problem addressed in this paper is an agent based distributed control. There is one agent in charge of each system partition and its duties are to negotiate the shared variables with other agents and to calculate the control actions from the MPC formulation of its partition.

Figure 1, on the left, shows a sample system divided into three partitions. There are three overlapping sets that contain four shared variables. The relations that represent those variables are shown on the right as lines. The problem consists in optimizing the manipulated variables of the global system in a distributed fashion, i.e. with three local control agents that must preserve consistency between the shared variables.

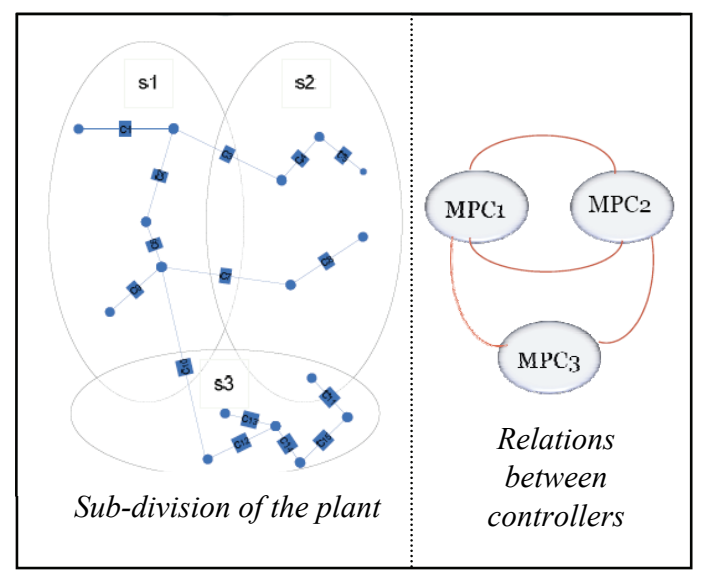

Figure 1: The problem of distributed control.

\section{DISTRIBUTED MPC}

In distributed control schemes, local control inputs are computed using local measurements and reduced-order models of the local dynamics (Scattolini, 2009).

Distributed MPC is attractive because it requires only local process data for controller design and model maintenance. Computing demands are smaller on each control agent. Furthermore, routine maintenance operations such as removing sensors or actuators for repairing are achieved much more easily under distributed MPC. Nevertheless, there is one well known drawback: the performance of distributed MPC is usually far from optimal when the subsystems interact significantly. Centralized MPC, on the other hand, achieves optimal nominal control for any system. However, centralized MPC is viewed by most practitioners as impractical and unsuitable for control of large networked systems. (Venkat et al., 2005).

Distributed MPC algorithms are classified into iterative and non-iterative and further subclassified into independent or cooperative algorithms.

In iterative algorithms information is bidirectionally transmitted among local regulators many times within the sampling time. In noniterative algorithms information is bi-directionally transmitted among local regulators only once within each sampling time. (Scattolini, 2009) gives a review of distributed control architectures for LSS.

Independent (non-cooperative) algorithms are widely studied in game theory. Their aim is to get better results than the other controllers, which are seen as opponents. They have also been applied in MPC distributed control strategies [see, for instance, (Jia \& Krogh, 2002) (Camponogara, 2002)].

Contrarily, cooperative algorithms intend to find a compromise for shared variables in order to maximize the performance of the complete system, worsening if necessary the performance of partitions. (Negenborn et al., 2008) and (Venkat et al., 2005) are two recent examples of the application of cooperative algorithms, the first one is noniterative and the second one is iterative.

\section{MULTI-AGENT MPC ARCHITECTURE}

In this section, the proposed multi-agent MPC (MAMPC) architecture is presented. First the elements of the proposed architecture are presented. Then, the whole architecture is described.

\subsection{Elements}

The elements of the proposed architecture are: $M P C$ Agents and Negotiator Agents. They interact over an Agent Platform that is composed by a set of Nodes. 
Definition 4. MPC Agent. An MPC Agent is the entity that is in charge of controlling one specific partition of the system.

There is one MPC Agent for each system partition. The MPC Agent solves an MPC control problem considering the internal variables of the partition and cooperating with one or more Negotiator Agents to determine the optimum value of the shared variables. $A$ is the set of MPC Agents

$$
A=\left\{a_{1}, a_{2}, \ldots, a_{n a}\right\}
$$

Definition 5. Negotiator Agent. A Negotiator Agent is the entity that is in charge of determining the value of one or more shared variables between two MPC Agents.

In this negotiation, each MPC Agent is arranged to cooperate so that the negotiator agent solves the optimization of a common goal by means of an algorithm based on Reinforcement Learning. A negotiator Agent exists for every pair of MPC Agents that have one or more shared variables in common. $N$ is the set of Negotiator Agents defined by

$$
N=\left\{n_{1}, n_{2}, \ldots, n_{n n}\right\}
$$

Definition 6. Nodes. A node is the physical device (commonly a computer) in which the agents are located. $W$ is the set of nodes defined by

$$
W=\left\{w_{1}, w_{2}, \ldots, w_{n w}\right\}
$$

There is a node for each MPC Agent. Nodes are communicated via some communication infrastructure (LAN, WAN or Internet).

Definition 7. Agent Platform. The agent platform works as a virtual machine providing the agents a homogenous medium to communicate and providing the user a way to manage agents. The agent platform is denoted by $b$.

This platform has to be installed and running in all nodes.

\subsection{Architecture}

Definition 8. MAMPC Architecture. The MAMPC distributed control architecture is defined as:

$$
\gamma=\left\{A, N, P, W, V_{n n}, U_{n a}, b\right\}
$$

where: $A$ is the set of MPC Agents, $N$ is the set of Negotiator Agents, $P$ is the system partitions, $W$ is the set of nodes, $V_{n n}$ is the set formed by all sets of Shared Variables, $U_{n a}$ is the set formed by all sets of Internal Variables and $b$ is the Agent platform.
A methodology has been developed to apply this MAMPC architecture in a given system. This methodology will be illustrated in the following section with an example.

\subsection{Cooperation of MPC-Agents}

The cooperative interaction of MPC agents is a basic issue in the proposed approach. Three main actions are necessary to perform this cooperation:

- To perform actions and provide data required by the Negotiatior Agent

- To accept the value(s) provided by the Negotiator Agent of its shared variable(s).

- To solve the MPC control problem of its partition, adjusting the value(s) of its shared control variable(s) in order to coordinate the solution of the negotiation.

The Negotiator Agent determines the optimal value of the values in set $V_{x}$. This set contains the shared variables of two, and just two MPC Agents. The Negotiator Agent optimizes them through a Negotiation algorithm based on Reinforcement Learning (RL). Each shared variable is an optimization problem. This problem is solved as a whole looking for the optimal value of the relation. The method is based on the reinforcements given at each step and on the experience obtained. This experience is stored in a knowledge base, one for each negotiation variable.

In the distributed model of the system, shared variables appear in the local models of each MPC Agent involved in the relation, therefore they end up duplicated.

The Negotiator Agent restores the broken connections when the system was partitioned, unifiying this duplicate variables in a single one, just as in the original model. Therefore, for the Negotiator Agent, this two control variables are taken as one.

The philosophy of the proposed negotiation algorithm is to consider the shared variables as belonging to a single problem with a single goal, instead of two different problems with conflicting goals. The Negotiator Agent solves the optimization problem for that variable and communicates the result to the MPC Agents at each sampling time. Then, MPC Agents set those values as a hard constraint in its respective internal control variables and recalculate the multivariable control problem.

The optimization of the Negotiator Agent algorithm is based on its experience and on maximizing the reinforcements received at every action taken in the past on similar situations. 
This algoritm is based on the Q-learning algorithm, and adapted to be applied in dynamical environments. Next, the formulation of the algorithm is detailed.

\subsubsection{Formulation of the Negotiation- learning Problem}

The goal of the Negotiator Agent is to detemine the optimal value of the set of shared variables $V_{x}$. Each element of the set $V_{x}$ is an optimization problem addressed individually by the Negotiator Agent and there is a knowledge base for each one. The internal architecture of the Negotiator Agent comprises the following elements:

- A set of knowledge bases (Q-tables); each Qtable represents the knowledge base of the agent, which has a Q-table for each shared variable because each one can have diferent behaviour and even different goals.

- A communication protocol that allows it to have bi-directional communication with two MPC-Agents.

- A negotiation algorithm

Next, these elements are described in further detail.

Q-Table. The Q-table represents the knowledge base of the agent, which has a Q-table for each shared variable because each one can have diferent behaviour and even different goals.

Q-tables maintain the reinforcement gained for each possible state and action. A state represents the global state of each sub-problem, which is established in terms of the error of the output with respect to the goal. The definition of the error that MPC Agents use is:

$$
\varepsilon_{i}=g_{i}-y_{i}
$$

where $\varepsilon_{i}$ is the error, $g_{i}$ is the goal and $y_{i}$ is the output of variable $i$.

The state value is determined by:

$$
S=\frac{\left|\varepsilon_{i 1}\right|+\left|\varepsilon_{i 2}\right|}{2}
$$

where $\varepsilon_{i l}$ is the error of the variable $i$ of first agent, and $\varepsilon_{i 2}$ of the corresponding variable in the second agent. This state is updated every sampling time.

Since states are continuous, they have to be discretized for the application of the RL algorithm.

Actions are all the posible values that the shared variable can take. Since actions are continuous, they have to be discretized for the application of the RL algorithm.
The reward function determines the reward of every action taken by the agent. In this case, the reward function is:

$$
r=\rho-s
$$

where $\rho$ is a value greater or equal than $s$

Communication Protocol. MPC Agents start the comunication by interchanging the resulting output of the control applied $\left(y_{i}(k)\right)$, the vector of controls applied $\left(u_{i}(k)\right)$, the absolute error whith respect to the goal of the shared variable $\varepsilon_{i}(k)$ and the sampling time $k$. Then, the algorithm of the Negotiator Agent is executed. When it finishes, it communicates the result of the optimization and the parameters needed by de MPC Agents to solve their multivariable problems, taking as restrictions the values given by the negotiator. Then the procces starts again.

Negotiation Algorithm. This algorithm is divided in two phases, the training phase and the exploitation phase. In both cases, the updated rule for Q-table values is:

$$
Q(s, a)=r+(\alpha \times Q(s, a))
$$

The training phase creates a new Q-table off-line using stored data obtained, for instance, from the control actions determined by the centralized approach.

Once the Q-table is initialized, the exploitation phase can start. The main difference here is that actions are chosen according to

$$
a^{\prime}=\max _{a}(Q(s, a))
$$

in order to select for the next time instant, the value of the action (negotiated variable) with maximum reward

\section{APPLICATION EXAMPLE}

\subsection{Description}

A small drinking water network is used to exemplify the proposed MAMPC architecture. The example was proposed in (Barcelli, 2008) where a centralized and a decentralized solution was studied and compared. This hypothetical water distribution network has 8 states (tanks) and 11 control variables (valves). It has been divided into two subsystems. Two MPC Agents are used to determine the internal control variables of each subsystem. Furthermore, one Negotiator Agent is responsible of negotiating 
the values of the two shared control variables between the two MPC agents (see Figure 2).

\subsection{Analysis}

In the analysis phase, the MAMPC Architecture is defined. This phase comprises the following tasks:

Definition of the Optimization Goals. The control goal of the application presented in Figure 2 is to keep a water volume in each tank around $3 \mathrm{~m}^{3}$.

Partitioning of the Network. The system is splitted into two partitions:

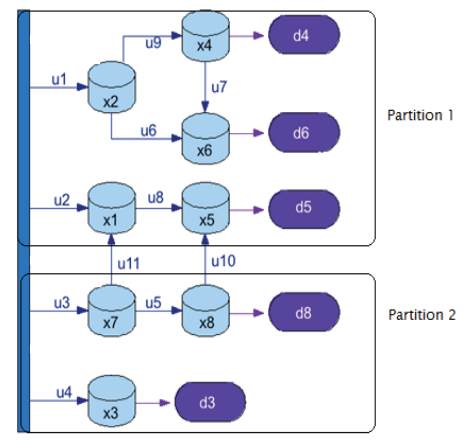

Figure 2: Case study and its partitioning.

$$
\begin{gathered}
p_{1}=\left\{x_{1}, x_{2}, x_{4}, x_{5}, x_{6}\right\} \\
p_{2}=\left\{x_{3}, x_{7}, x_{8}\right\} \\
V=\left\{u_{10}, u_{11}\right\} \\
U_{1}=\left\{u_{1}, u_{2}, u_{6}, u_{7}, u_{8}, u_{9}\right\} \\
U_{2}=\left\{u_{3}, u_{4}, u_{5}\right\}
\end{gathered}
$$

The plant is defined by all its state and input variables

$$
\begin{gathered}
\text { Plant }=\left\{x_{1}, x_{2}, x_{3}, x_{4}, x_{5}, x_{6}, x_{7}, x_{8}, u_{1}, u_{2},\right. \\
\left.u_{3}, u_{4}, u_{5}, u_{6}, u_{7}, u_{8}, u_{9}, u_{10}, u_{11}\right\}
\end{gathered}
$$

An important step is to check that the partinioning of the plant leads to a complete set of partitions. This is accomplished verifying the following relation:

$$
\text { Plant }=P \cup U \cup V
$$

which can be easily verified,

$$
\text { Plant }=p_{1} \cup p_{2} \cup U_{1} \cup U_{2} \cup V
$$

Thus, the partition is a complete set of partitions.

Definition of the Architecture. In this step, the MAMPC Architecture is defined for the water network case study. Considering the definition of the architecture in (8), the remaining elements are defined as follows:

$$
\begin{gathered}
A=\left\{a_{1}, a_{2}\right\} \\
N=\left\{n_{1}\right\} \\
W=\left\{w_{1}, w_{2}\right\}
\end{gathered}
$$

Inclusion of Restrictions and Considerations. The maximum water volume in tanks is $20 \mathrm{~m}^{3}$, the control value of the measured variables ranges from 0.0 to 0.4 except for $u_{2}$ that it ranges from 0.0 to 0.1 . The sampling time is 1 hour and the prediction horizon is 24 hours. The demands are considered as measured perturbations. They typically present a sinusoidal behaviour throghout the day.

\subsection{Design}

In the design process, the subproblems of every MPC Agent and Negotiator Agent are formulated. This formulation is based on the information collected in the analysis phase that allows to know the internal structure of the MPC Agents.

The core of the MPC agent is a MPC controller. This controller solves the multivariable problem of one partition of the plant based on a model. This model contains the set $u_{x}$ of the agent. Other important part of the MPC Agent is the communication block. MPC Agents can communicate in a sophisticated way because they are implemented using the Agent Oriented Paradigm. This paradigm provides methods, standards and tools that allow good communication skills.

Formulation of the MPC Problem. In this step, all the MPC parameters and requierements have to be defined for both agents, such as:

1)The plant; 2) The measured, non-measured and manipulated variables; 3) Limits and constraints; 4) The negotiation variables are set as restrictions; 5) References (goals); 6) Prediction horizon; 7) Control Horizon; 8) Initial state; 9) Perturbations models

All these data have to be set in all MPC Agents. The prediction and control horizon should be the same for all MPC Agents.

\subsubsection{Training and Exploitation}

As in any RL algorithm, the proposed architecture is based on the agent experience and the expected reinforcements. The richer the agent experience has been, the more efficient the optimization algorithm will be. 
An off-line training was done in order to provide this experience to the Negotiatior Agent. First, control actions determined from a 48 hours scenario of the centralized approach were used as initialization values for the agent training process. From this point, the training continued taking random actions The reward was calculated for all actions.

In the RL exploitation phase the knowledge adquired in the exploration (and training) phase is used.

The exploitation phase uses the knowledge adquired in order to solve the MPC distributed problem through the MA system.

\section{RESULTS}

The results obtained using the proposed MAMPC Architecture are shown in Figure 3. Each graph presents a 48 hour scenario, showing the absolute error with respect to the goal (volume of $3 \mathrm{~m}^{3}$ in each tank) at every time step. The results are contrasted with the centralized solution (dashed line) for all tanks.

The distributed solution was expected not to be as good as the centralized one. However, the graphs shows that, in some cases (tanks 1, 2, 7 and 8) the distributed MAMPC Architecture solution is better. It is important to note that the volume of tanks 1,7 and 8 depends on the valute of the negotiated variables $\left(u_{10}\right.$ and $\left.u_{11}\right)$.

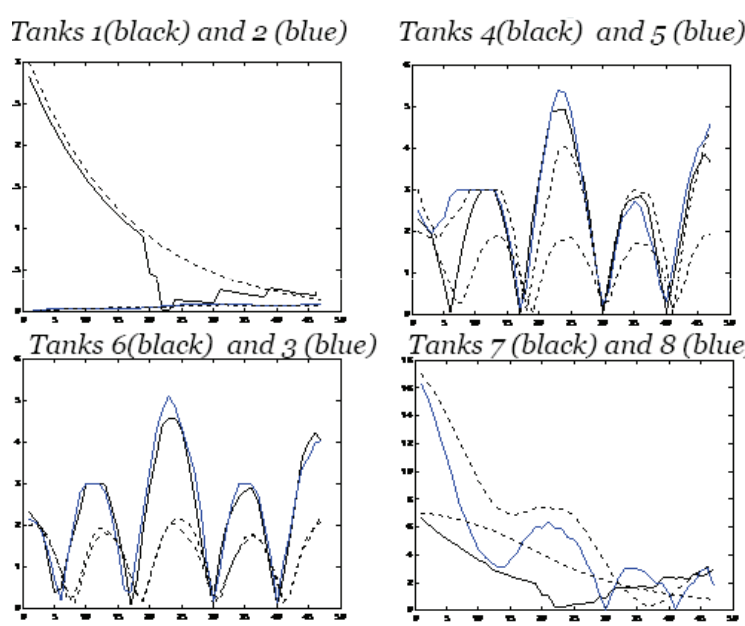

Figure 3: Distributed MA-MPC Architecture solution against centralized solution.

\section{CONCLUSIONS}

The results obtained suggest that the use of a MultiAgent control architecture based on negotiation can converge to the centralized solution with an acceptable degree of approximation but taking advantage from the MAS properties and the tools that the Agent Oriented Paradigm (AOP) provides for development and implementation. Even more, the application of learning techniques can provide the Negotiatior Agent the ability of prediction. Training of the negotiator can be made directly from a centralized MPC or from human operator driven control. In order to achieve optimization, no model is needed by the negotiator. Data from centralized MPC is advisable but not essential. The type and quality of the training is a very important issue in order to obtain an efficient optimization. Also the compromise between exploration and explotation can be implemented on line to enable the system not just adaptation to the problem but adaptation to changes in time. In this paper, this capability is not addressed in training but in exploring during the optimization. Communication protocols and coordination methods for MAS have to be studied and tested in a more complex case of study in which many agents interact.

\section{FURTHER RESEARCH}

The MAMPC Architecture presented in this work is currently being tested on the Barcelona water transport network. The Barcelona water network is comprised of 200 sectors with approximately 400 control points. At present, the Barcelona information system receives, in real time, data from 200 control points, mainly through flow meters and a few pressure sensors. This network have been used as a LSS case of study to test several LSS control approaches, see (Brdys \& Ulanicki 1994) and (Cembrano et al, 2002) (Cembrano et al, 2004) (Cembrano et al., 2000). As starting point for the application of the MAMPC Architecture, recent work on centralized (Caini et al., 2009) and decentralized MPC (Fambrini \& Ocampo, 2009) applied to the Barcelona network is being used, as well as, the partitioning algorithm developed by (Barcelli, 2008). 


\section{ACKNOWLEDGEMENTS}

This work is in the context of the European Project Decentralized and Wireless Control of Large Scale Systems, WIDE - 224168 - FP7-ICT-2007-2. First author is sponsored by the Consejo Nacional de Ciencia y Tecnología (CONACYT) of México and is partially supported by the Instituto Tecnológico Superior de Cajeme (ITESCA).

\section{REFERENCES}

Barcelli, D. (2008). Optimal decomposition of Barcelona's water distribution network system for applying dsitribuited Model Predictive Control. Master thesis . Universitat Politècnica de Cataluña-IRI-Universitá degli Study di Siena.

Bemporad, A. and Morari, M. "Robust model predictive control: A survey," in Robustness in Identification and Control (Lecture Notes in Control and Information Sciences), vol. 245. New York: Springer-Verlag, 1999, pp. 207-226.

Brdys, M. A., \& Ulanicki, B. (1994). Operational control of water systems, Structures, Algorithms and Applications. Great Britain: Prentice Hall International.

Caini, E., Puig Cayuela, V., \& Cembrano, G. (2009). Development of a simulation environmet for water drinking networks: Application to the validation of a centralized MPC controller for the Barcelona Case of study. Barcelona, Spain: IRI-CSIC-UPC.

Camacho, E. F., \& Bordons, C. (2004). Model Predictive Control. Springer-Verlag, London.

Cembrano, G., Figueras, J., Quevedo, J., Puig, V., Salamero, M., \& Martí, J. (2002). Global control of the Barcelona Sewerage system for environment protection. IFAC.

Cembrano, G., Quevedo, J., Salamero, M., Puig, V., Figueras, J., \& Martí, J. (2004). Optimal control of urban drainage systems. A case of study. Control Engineering Practice (12), 1-9.

Cembrano, G., Wells, G., Quevedo, J., Pérez, R., \& Argelaguet, R. (2000). Optimal Control of a water distribution network in a supervisory control system. Control of Engineering Practice (8), 1177-1188.

Fambrini, V., \& Ocampo Martinez, C. (2009). Modelling a decentralized Model Predictive Control of drinking water network. Barcelona, Spain: IRI-CSIC-UPC.

Negenborn, R. R. (2008). Multi-Agent Model Predictive Control with applications to power networks. Engineering Applications of Artificial Intelligence , 21, 353-366.

Negenborn, R. R., De Shutter, B., \& Hellendoorn, J. (2004). Multi-Agent model predictive control: A survey. Technical report, Delf University of Technology, Delf center for systems and control.
Qin, S. J., \& Badwell, T. A. (2003). A survey of industrial Model Predictive Control Technology. Control Engineering Practice, 11, pp. 733-764.

Scattolini, R. (2009). Architectures for distributed and hiearical Model Predictive Control- A Review. Journal of Process Control , 723-731.

Siljack, D.D. (1991). Decentralized Control of Complex Systems, Academic Press, New York.

Venkat, A. N., Rawlings, J. B., \& Wrigth, S. J. (2005). Stability and Optimality of distributed Model Predictive Control. IEEE Conference on Decision and Control / IEE European. 